\title{
Analytical Challenges in Development of Chemoresistance Predictors for Precision Oncology
}

\author{
Mariana Bleker de Oliveira, ${ }^{1}$ Vasilij Koshkin, ${ }^{1}$ Geoffrey Liu, ${ }^{2}$ and Sergey N. Krylov ${ }^{1}$ \\ ${ }^{1}$ Department of Chemistry and Centre for Research on Biomolecular Interactions, York University, \\ Toronto M3J 1P3, Canada \\ ${ }^{2}$ Department of Medicine, Medical Oncology, Princess Margaret Cancer Centre, Toronto M5G 2M9, \\ Canada
}

\begin{abstract}
Chemoresistance, i.e., tumor insensitivity to chemotherapy, shortens life expectancy of cancer patients. Despite the availability of new treatment options, initial systemic regimens for solid tumors are dominated by a set of standard chemotherapy drugs, and alternative therapies are used only when a patient has demonstrated chemoresistance clinically. Chemoresistance predictors use laboratory parameters measured on tissue samples to predict patient's response to chemotherapy and help to avoid application of chemotherapy to chemoresistant patients. Despite thousands of publications on putative chemoresistance predictors, there are only about a dozen predictors that are sufficiently accurate for precision oncology. One of the major reasons for inaccuracy of predictors is inaccuracy of analytical methods utilized to measure their laboratory parameters: an inaccurate method leads to an inaccurate predictor. The goal of this study was to identify analytical challenges in chemoresistance-predictor development and suggest ways to overcome them. Here we describe principles of chemoresistance predictor development via correlating a clinical parameter, which manifests disease state, with a laboratory parameter. We further classify predictors based on the nature of laboratory parameters and analyze advantages and limitations of different predictors using the reliability of analytical methods utilized for measuring laboratory parameters as a criterion. Our eventual focus is on predictors with known mechanisms of reactions involved in drug resistance (drug extrusion, drug degradation, and DNA damage repair) and using rate constants of these reactions to establish accurate and robust laboratory parameters. Many aspects and conclusions of our analysis are applicable to all types of disease biomarkers built upon the correlation of clinical and laboratory parameters.
\end{abstract}

\section{TABLE OF CONTENTS}

\section{Section}

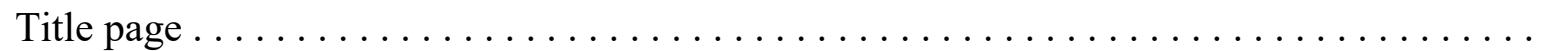

Note S1: Current definitions of precision, accuracy, robustness and ruggedness by regulatory

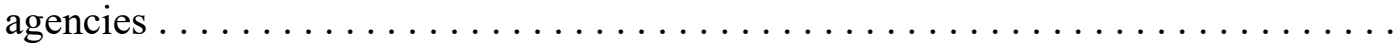

Note S2: Consideration of effectiveness of alternative therapy $\ldots \ldots \ldots \ldots \ldots \ldots \ldots \ldots$

Table S1: Predictive biomarkers of cancer approved by FDA for clinical use . . . . . . . S-5

Figure S1: Schematic representation of Cytometry of Reaction Rate Constant . . . . . . . S-6

Figure S2: Three molecular mechanisms of chemoresistance $\ldots \ldots \ldots \ldots \ldots \ldots \ldots \ldots$

Supporting References $\ldots \ldots \ldots \ldots \ldots \ldots \ldots \ldots \ldots \ldots \ldots \ldots \ldots \ldots \ldots \ldots$ 


\section{Note S1: Current definitions of precision, accuracy, robustness and ruggedness by regulatory agencies}

Precision is defined as "the closeness of agreement (degree of scatter) between a series of measurements obtained from multiple sampling of the same homogeneous sample under the prescribed conditions". ${ }^{1}$ Accuracy is defined as "the closeness of agreement between the value which is accepted either as a conventional true value or an accepted reference value and the value found". ${ }^{1}$ The robustness of an analytical procedure is defined as "a measure of its capacity to remain unaffected by small, but deliberate variations in method parameters and provides an indication of its reliability during normal usage". ${ }^{1}$ Ruggedness of an analytical method is defined as "the degree of reproducibility of test results obtained by the analysis of the same sample under a variety of normal test conditions, such as different laboratories, different analysts, different instruments, different lots of reagents, different elapsed assay times, different assay temperatures, different days, etc.". ${ }^{2}$ 


\section{Note S2: Consideration of effectiveness of alternative therapy}

Deciding to replace an established frontline chemotherapy with an alternative therapy in an individual patient requires that chemoresistance be very reliably predicted. False-positive results in a chemoresistance test may lead to unjustified replacement of chemotherapy with a less effective alternative therapy for non-chemoresistant (i.e. chemosensitive) patients. ${ }^{3}$ According to the definition of chemosensitivity, the effectiveness of frontline chemotherapy $\left(E_{\text {chemo }}\right)$ is equal to unity for chemosensitive patients: $E_{\text {chemo }}=1$. The effectiveness of the alternative therapy ( $\left.E_{\text {alt }}\right)$ is lower than $E_{\text {chemo }}$ for chemosensitive patients, e.g. $E_{\text {alt }}<1$. On the other hand, $E_{\text {alt }}$ can be presumed to be the same for chemoresistant and chemosensitive patients as the alternative therapy has a mechanism of action different from the cytotoxicity mechanism of the first line chemotherapeutic agent. The value of $E_{\text {alt }}$ will define the required quality of a chemoresistance test. Two parameters of a chemoresistance-predictor test define its quality: sensitivity and specificity. Sensitivity of a chemoresistance predictor is defined as a fraction of patients who are correctly predicted to be chemoresistant among those who are truly chemoresistant, and thus should be treated with the alternative therapy. Specificity, in turn, is a fraction of patients who are correctly predicted to be chemosensitive among those who are truly chemosensitive, and who should be treated with chemotherapy. ${ }^{4}$ The requirement for a predictor test is that for chemosensitive patients, $E_{\text {alt }}$ should be higher than the negative likelihood ratio, which is a ratio between the probability of false-detection of chemoresistance (1 - assay sensitivity) and that of correctly ruling out chemoresistance (sensitivity), ${ }^{5}$ which can be summarized as:

$E_{\text {alt }}>(1-$ sensitivity $) /$ specificity

Values for assay sensitivity and specificity can vary between 0 and 1 . When applying real values of alternative therapy effectiveness $\left(E_{\text {alt }}\right)$ to the above inequality, the need for high sensitivity and specificity becomes evident. For example, for metastatic colorectal cancer patients, the effectiveness of second-line therapy is $0.61 ;^{6}$ therefore, assay sensitivity and specificity must satisfy inequality $(1-$ sensitivity $) /$ specificity $<0.61$. When sensitivity is set at 1 , the numerator is equal to zero, and any specificity value would make the statement true. In contrast, when specificity is set at 1 , sensitivity needs to be higher than 0.39 to satisfy the inequality. In another example, for renal cell carcinoma patients, the effectiveness of second-line therapy is only $0.13 ;^{7}$ therefore, assay sensitivity and specificity must satisfy inequality $(1-$ sensitivity $)$ /specificity $<0.13$. Again, for sensitivity of 1 , any specificity value would be sufficient; however, when specificity is set at 1 , sensitivity should be higher than 0.87 . The above examples are solely to illustrate how the effectiveness of an alternative therapy imposes requirements on the sensitivity and specificity of a chemoresistance-predictor test. Higher values of $E_{\text {alt }}$ allow more tolerance for assay sensitivity while low values of $E_{\text {alt }}$ impose very strict requirements on this characteristic. Assay specificity is a less restrictive parameter, but it is also important as low specificity must be compensated by the increase in sensitivity. In clinical practice, the actual threshold set for the negative likelihood ratio may also be influenced by factors other than treatment effectiveness: issues such as cost effectiveness, toxicity of chemotherapy versus alternative regimen, and patient preference may supersede or alter the specific thresholds; however, we will take the purist approach, assuming that all other non-effectiveness factors are equal between treatment options.

It is important to emphasize that diagnostic sensitivity and specificity of a chemoresistance predictor depend on the choice of the threshold which divides cases presumed to be chemoresistant and chemosensitive (horizonal line in Figure 2 in the main text). When the threshold is lowered, more truechemoresistant cases are identified at the expense of a growing number of false-chemoresistant cases. Thus, lowering the threshold increases the sensitivity but decreases the specificity. A plot of sensitivity versus 1 - specificity is called receiving operating characteristic (ROC) curve, which can evaluate the diagnostic ability of a test to truly discriminate between the two different states of the patients, ${ }^{8}$ i.e. chemoresistance or chemosensitivity. The area under the curve (AUC) is an important performance characteristic of the predictor, and it can be interpreted as the average value of sensitivity for all possible values of specificity. ${ }^{8}$ AUC $=1.0$ indicates perfect separability of chemoresistance from 
chemosensitivity and corresponds to an ideal predictor. A predictor with $\mathrm{AUC}=0.5$ cannot separate chemoresistance from chemosensitivity, meaning that the predictor test yields chemoresistant or chemosensitive classifications at chance, without any correlation with the true status of the patient. ${ }^{9}$

If a reliable chemoresistance predictor is available, a test for chemoresistance should take place before the first round of treatment for every patient considered for chemotherapy. Such a test should also be used before the subsequent rounds of treatment for patients who did not show pre-existing chemoresistance but had eventual cancer progression (i.e. tumor growth or development of new metastasis) at any time point after the start of therapy. ${ }^{10}$ Chemoresistance prediction, if reliable, is thus envisioned to benefit patients with both pre-existing and acquired resistance. 
Table S1: Predictive biomarkers of cancer approved by FDA for clinical use.*

\begin{tabular}{|c|c|c|}
\hline Predictor classification & Year*** & Cancer type \\
\hline \multicolumn{3}{|l|}{ Genetic aberrations (11) } \\
\hline$A L K$ (gene rearrangement)** & 2010 & NSCLC \\
\hline$B R A F$ (mutation) & 2010 & Melanoma \\
\hline$B R C A$ (mutation)** & 2011 & Breast; ovarian \\
\hline BCR-ABL (chromosome translocation) & 2001 & $\mathrm{CML}$ \\
\hline$c-K I T$ (mutation) & 2002 & GIST \\
\hline$E G F R$ (mutation)** & 2010 & NSCLC \\
\hline$I D H$ (mutation) & 2013 & Glioma \\
\hline$K R A S$ (mutation)** & 2008 & NSCLC; CRC \\
\hline PDGFR (mutation) & 2002 & CML; GIST \\
\hline PML-RAR (chromosome translocation) & 2001 & APL \\
\hline ROSI (gene rearrangement)** & 2017 & NSCLC \\
\hline \multicolumn{3}{|l|}{ Quantities of molecules (6) } \\
\hline EGFR (protein expression)** & 2004 & $\mathrm{CRC}$ \\
\hline ER (protein expression)** & 1977 & Breast \\
\hline HER2 (protein expression)** & 1998 & Breast \\
\hline HER2 (gene amplification)** & 1998 & Breast \\
\hline PDL-1 (protein expression)** & 2015 & NSCLC; melanoma; bladder \\
\hline $\begin{array}{l}\text { MSI-H (alteration in the number of } \\
\text { repeated DNA bases in microsatellites)** }\end{array}$ & 2017 & $\begin{array}{l}\text { Adult and pediatric unresectable } \\
\text { or metastatic solid tumors with } \\
\text { the biomarker. }\end{array}$ \\
\hline
\end{tabular}

Abbreviations: ER, estrogen receptor; MSI-H, microsatellite instability high; NSCLC, nonsmall-cell lung cancer; CML, chronic myeloid leukemia; GIST, gastrointestinal stromal tumors; CRC, colorectal cancer; APL, acute promyelocytic leukemia.

* Prognostic biomarkers such as Mammaprint or Oncotype Dx that are not tied to the effectiveness of specific systemic therapies are not included in the table.

** Chemoresistance predictors.

*** Year in which the biomarker was either approved by FDA (with or without an accompanying drug) or was implemented for clinical use. 


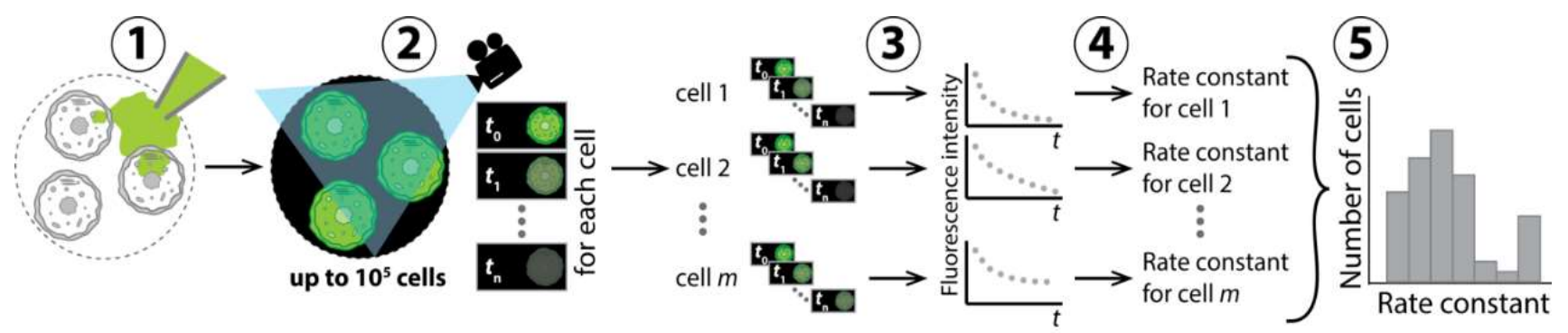

Figure S1: Schematic representation of Cytometry of Reaction Rate Constant. 1) A fluorescent substrate is added to the cells. 2) The reduction in cellular fluorescence intensity as a result of the catalytic reaction is monitored over time as sequential images are captured. 3 and 4) Individual reaction rate constants are determined for each single cell. 5) The values of rate constants are plotted on a "number of cells vs. rate constant" histogram to determine cell-population heterogeneity. Adapted from Koshkin et al., Anal. Chem. 2019, 91, 4186-4194. 


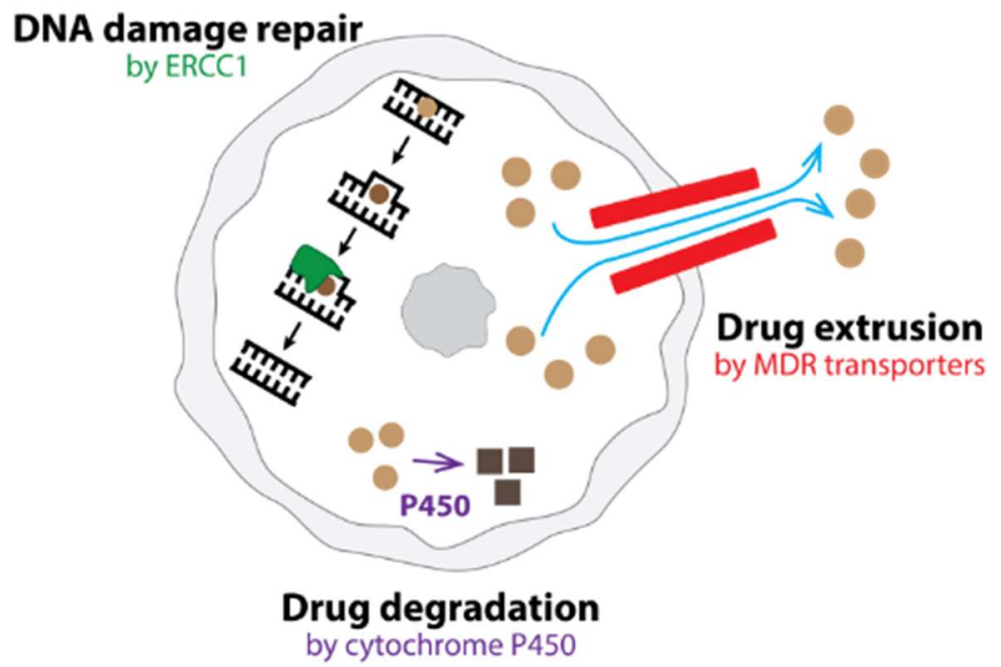

Figure S2. Three molecular mechanisms of chemoresistance. The three mechanisms directly related to the chemotherapeutic agent include: drug extrusion by MDR transporters, drug degradation by cytochrome P450, and repair of drug-induced DNA damage by ERCC1. 


\section{Supporting References}

(1) Validation of analytical procedures: text and methodology Q2(R1). International conference on harmonisation of technical requirements for registration of pharmaceuticals for human use, 2005.

(2) USP 42-NF 37. In Supplement 1: February 2019. Supplement 2: June 2019.: United States Pharmacopeia Convention Inc., 2018.

(3) Pletcher, M. J.; Pignone, M. Evaluating the clinical utility of a biomarker: a review of methods for estimating health impact. Circulation 2011, 123, 1116-1124.

(4) Janes, H.; Pepe, M. S.; McShane, L. M.; Sargent, D. J.; Heagerty, P. J. The Fundamental Difficulty With Evaluating the Accuracy of Biomarkers for Guiding Treatment. J. Natl. Cancer Inst. 2015, 107, djv157.

(5) Attia, J. Diagnostic tests: Moving beyond sensitivity and specificity: Using likelihood ration to help interpret diagnostic tests. Aust. Prescr. 2003, 26, 111-113.

(6) Bazarbashi, S.; Hakoun, A. M.; Gad, A. M.; Elshenawy, M. A.; Aljubran, A.; Alzahrani, A. M.; Eldali, A. Efficacy of second-line chemotherapy after a first-line triplet in patients with metastatic colorectal cancer. Curr. Oncol. 2019, 26, e24-e29.

(7) Herbst, R. S.; Soria, J. C.; Kowanetz, M.; Fine, G. D.; Hamid, O.; Gordon, M. S.; Sosman, J. A.; McDermott, D. F.; Powderly, J. D.; Gettinger, S. N.; Kohrt, H. E.; Horn, L.; Lawrence, D. P.; Rost, S.; Leabman, M.; Xiao, Y.; Mokatrin, A.; Koeppen, H.; Hegde, P. S.; Mellman, I., et al. Predictive correlates of response to the anti-PD-L1 antibody MPDL3280A in cancer patients. Nature 2014, 515, 563-567.

(8) Hanley, J.A; McNeil, B.J. The meaning and use of the area under a receiver operating characteristic (ROC) curve. Radiology 1982,143, 29-36.

(9) Hajian-Tilaki, K. Receiver Operating Characteristic (ROC) Curve Analysis for Medical Diagnostic Test Evaluation. Caspian J. Intern. Med. 2013, 4, 627-635.

(10) Perrone, M. G.; Luisi, O.; De Grassi, A.; Ferorelli, S.; Cormio, G.; Scilimati, A. Translational Theragnostic of Ovarian Cancer: Where do we stand? Curr. Med. Chem. 2019 published online ahead of print. 